Research Article

\title{
Interval-Valued Complex Fuzzy Geometric Aggregation Operators and Their Application to Decision Making
}

\author{
Songsong Dai $\mathbb{D}^{1}{ }^{1}$ Lvqing $\mathrm{Bi}^{2}{ }^{2}$ and $\mathrm{Bo} \mathrm{Hu}^{3}$ \\ ${ }^{1}$ School of Electronics and Information Engineering, Taizhou University, Taizhou 318000, China \\ ${ }^{2}$ School of Electronics and Communication Engineering, \\ Guangxi Colleges and Universities Key Laboratory of Complex System Optimization and Big Data Processing, \\ Yulin Normal University, Yulin 537000, China \\ ${ }^{3}$ School of Big Data and Computer Science, Guizhou Normal University, Guiyang 550025, China
}

Correspondence should be addressed to Songsong Dai; ssdai@stu.xmu.edu.cn

Received 29 May 2020; Accepted 3 July 2020; Published 22 July 2020

Guest Editor: Zaoli Yang

Copyright $(2020$ Songsong Dai et al. This is an open access article distributed under the Creative Commons Attribution License, which permits unrestricted use, distribution, and reproduction in any medium, provided the original work is properly cited.

This paper investigates the geometric aggregation operators for aggregating the interval-valued complex fuzzy sets (IVCFSs) whose membership grades are a special set of complex numbers. We develop some geometric aggregation operators under the interval-valued complex fuzzy environment, namely, interval-valued complex fuzzy geometric (IVCFG), interval-valued complex fuzzy weighted geometric (IVCFWG), and interval-valued complex fuzzy ordered weighted geometric (IVCFOWG) operators. Then, we investigate the rotational and reflectional invariances of these operators. Further, a decision-making approach based on these operators is presented under the interval-valued complex fuzzy environment and an example is illustrated to demonstrate the efficiency of the proposed approach.

\section{Introduction}

The aggregation operator is a powerful method for decision making, pattern recognition, and cluster analysis. In the past decades, in both theoretical and applied studies, aggregation operators have attained great advances. Many types of aggregation operators have been proposed under different environments, such as fuzzy environment [1-4], intuitionistic fuzzy environment [5-9], interval-valued intuitionistic fuzzy environment [10-14], Pythagorean fuzzy environment [15-17], neutrosophic fuzzy environment [18-20], and hesitant fuzzy environment [21-25].

In the above fuzzy environments, membership degrees are the subsets of real numbers. As a generalization of traditional fuzzy set [26], Ramot et al. [27] introduced the concept of complex fuzzy set (CFS), which is characterized by a complex-valued membership function. In many practical situations, complex fuzzy sets are useful [28-40]. Moreover, many researchers extended the concept of CFS to interval-valued complex fuzzy set (IVCFS) [41, 42] and complex intuitionistic fuzzy set (CIFS) [43]. Therefore, many researchers discussed how to aggregate CFSs. Ramot et al [32] introduced the concept of complex fuzzy aggregation. $\mathrm{Ma}$ et al. [38] proposed a product-sum aggregation operator under complex fuzzy environment. Bi et al. $[39,40]$ proposed several aggregation operators under complex fuzzy environment. Garg and Rani $[44,45]$ investigated the aggregation operators under complex intuitionistic fuzzy environment.

However, we still have the key question: why complex fuzzy aggregation? Moreover, why complex fuzzy sets? As mentioned in [46], from mathematical and practical viewpoints, complex fuzzy sets are natural and useful. But complex fuzzy sets (CFSs) remain a puzzle from the intuitive viewpoint. Fuzzy sets and other extensions give intuitively clear way to describe how humans deal with different types of uncertainty. So before we start to examine the information aggregation issue under interval-valued complex fuzzy environment, we first discuss some phenomena which maybe ignored in real life. When we ask the way, two persons may 
give the answers "it is about $1 \mathrm{~km}$ away," and then we think that $1 \mathrm{~km}$ is a reasonable result. However, if their answers are not exclusively same about direction, as shown in Figure 1, $0.95 \mathrm{~km}$ also is a reasonable result since $C=(A+B) / 2$.

How does this phenomenon affect human decision making? For example, there are two hospitals $H_{1}$ and $H_{2}$; which one is the nearest hospital? Then, we get data about distance and direction from strangers. It is a very interesting case; two strangers both agree that hospital $H_{1}$ is nearer than hospital $\mathrm{H}_{2}$, but after data aggregation, the result that $\mathrm{H}_{2}$ is nearer than hospital $H_{1}$ is also reasonable, as shown in Figure 2. The order only relying on the distance is reasonable since we want to go to the nearest hospital. The method based on complex fuzzy aggregation is reasonable since it is a center-based method.

Complex fuzzy aggregation operator can perfectly describe above phenomenon in human decision making since it does not satisfy the property of amplitude monotonicity [40]. Monotonicity is a basic property, which holds in many types of aggregation operators under different fuzzy environments [1-25]. Complex fuzzy aggregation operator as a nonmonotone average is very natural and gives an intuitive way to describe how humans deal with such type of uncertainty.

In this paper, we focus on the aggregation operator under interval-valued complex fuzzy environment. IVCFSs also are very appropriate for some applications in real life. For example, when we get lost, we often ask strangers for directions in our daily life. Then, we get some answers, such as " $0.5-0.6 \mathrm{~km}$, east" and " $0.5-0.7 \mathrm{~km}$, northeast." These answers can be represented in terms of IVCFSs. We present the theory of the weighted geometric aggregation operators among the IVCFSs. First we review necessary concepts and some basic properties related to this paper in Section 2. In Section 3, we present an interval-valued complex fuzzy weighted geometric (IVCFWG) operator on CFSs. In Section 4 , we present an interval-valued complex fuzzy ordered weighted geometric (IVCFOWG) operator. In Section 5, we present a decision-making approach based on the proposed operator under IVCFS environment. Finally, conclusions are given in Section 6.

\section{Preliminaries}

In this paper, our discussion is based on interval-valued complex fuzzy set theory. Some basic concepts are recalled below, whereas for other concepts, refer to reports from Ref. [27, 28, 41, 42, 47].

Let $D$ be the set of complex numbers on complex unit disk, i.e., $D=\{a \in \mathbb{C}|| a \mid \leq 1\}$. Let $U$ be a fixed universe, and a mapping $A: U \longrightarrow D$ is called a complex fuzzy set on $U$.

Let $\mathscr{I}^{[0,1]}$ be the set of all closed subintervals of $[0,1]$, i.e., $\mathscr{I}^{[0,1]}=\{[a, b] \mid 0 \leq a \leq b \leq 1\}$. Let $\dot{D}$ be the boundary set of $D$ i.e., $\dot{D}=\{a \in \mathbb{C}|| a \mid=1\}$. A mapping $A: U \longrightarrow \mathscr{I}^{[0,1]} \cdot \dot{D}$ is called an IVCFS on $U$. For any $x \in U$, its membership degree $\mu_{A}(x)$ is

$$
\left[\underline{r_{A}(x)}, \overline{r_{A}(x)}\right] \cdot e^{j v_{A}(x)},
$$

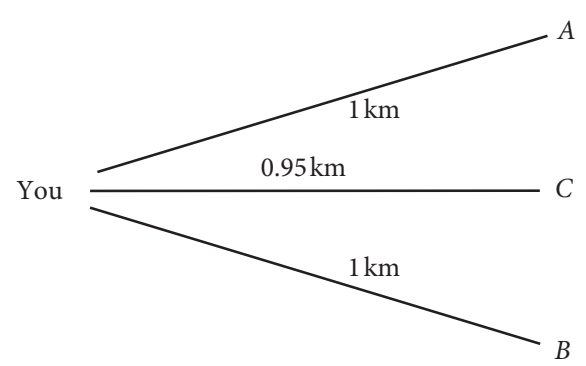

FIgUre 1: A target location example.

where $j=\sqrt{-1}, \mathscr{I}^{[0,1]} \cdot \dot{D}$ is the dot product set of $\mathscr{I}^{[0,1]}$ and $\dot{D},\left[r_{A}(x), \overline{r_{A}(x)}\right] \in \mathscr{I}^{[0,1]}$ is the interval-valued amplitude part, and $v_{A}(x) \in \mathbb{R}$ is the phase part.

For convenience, we only consider the values on $\mathscr{I}^{[0,1]} \cdot \dot{D}$, which are called interval-valued complex fuzzy values (IVCFVs). Let $a=\left[\underline{r_{a}}, \overline{r_{a}}\right] \cdot e^{j v_{a}}$ be an IVCFV, where the interval-valued amplitude part is $\left[r_{a}, \overline{r_{a}}\right] \in \mathscr{I}^{[0,1]}$ and the phase part is $v_{a} \in \mathbb{R}$. The modulus of $a$ is the intervalvalued amplitude part $\left[r_{a}, \overline{r_{a}}\right]$, denoted by $|a|$. An order of IVCFVs is defined by the interval-valued amplitude part, i.e.,

$$
|a| \leq|b| \text { if } \underline{r_{a}} \leq \underline{r_{b}} \text { and } \overline{r_{a}} \leq \overline{r_{b}} \text {. }
$$

Let $a=\left[r_{a}, \overline{r_{a}}\right] \cdot e^{j v_{a}}$ and $b=\left[r_{b}, \overline{r_{b}}\right] \cdot e^{j v_{b}}$ be two IVCFVs and let the parameters be $\overline{\lambda>0}$ and $\theta \in \mathbb{R}$; four operators of IVCFVs include multiplication, power, rotation, and reflection which are defined as follows.

(i) Multiplication of two IVCFVs $a, b \in \mathscr{I}^{[0,1]} \cdot \dot{D}$ :

$$
a \otimes b=\left[\underline{r_{a}} \cdot \underline{r_{b}}, \overline{r_{a}} \cdot \overline{r_{b}}\right] \cdot e^{j\left(v_{a}+v_{b}\right)} .
$$

(ii) Power of an IVCFV $a \in \mathscr{I}^{[0,1]} \cdot \dot{D}$ :

$$
a^{\lambda}=\left[\underline{\lambda} \underline{r_{a}}, \overline{r_{a}} \lambda\right] \cdot e^{j \lambda v_{a}} .
$$

(iii) Rotation of an IVCFV $a \in \mathscr{I}^{[0,1]} \cdot \dot{D}$ :

$$
\operatorname{Rot}_{\theta}(a)=\left[\underline{r_{a}}, \overline{r_{a}}\right] \cdot e^{j\left(v_{a}+\theta\right)}
$$

(iv) Reflection of an IVCFV $a \in \mathscr{I}^{[0,1]} \cdot \dot{D}$ :

$$
\operatorname{Ref}(a)=\left[\underline{r_{a}}, \overline{r_{a}}\right] \cdot e^{-j v_{a}} .
$$

When $a, b \in D, \otimes$ is a complex intersection defined by Ramot et al. [27]. When $a, b \in[0,1], \otimes$ is a t-norm [48].

Theorem 1. Suppose that $a, b, c$ are three IVCFVs, and the parameters are $\lambda_{1}>0, \lambda_{2}>0$, and $\theta_{1}, \theta_{2} \in \mathbb{R}$. Then, we have

(1) $a \otimes b=b \otimes a$

(2) $(a \otimes b) \otimes c=a \otimes(b \otimes c)$

(3) $(a \otimes b)^{\lambda_{1}}=a^{\lambda_{1}} \otimes b^{\lambda_{1}}$ 


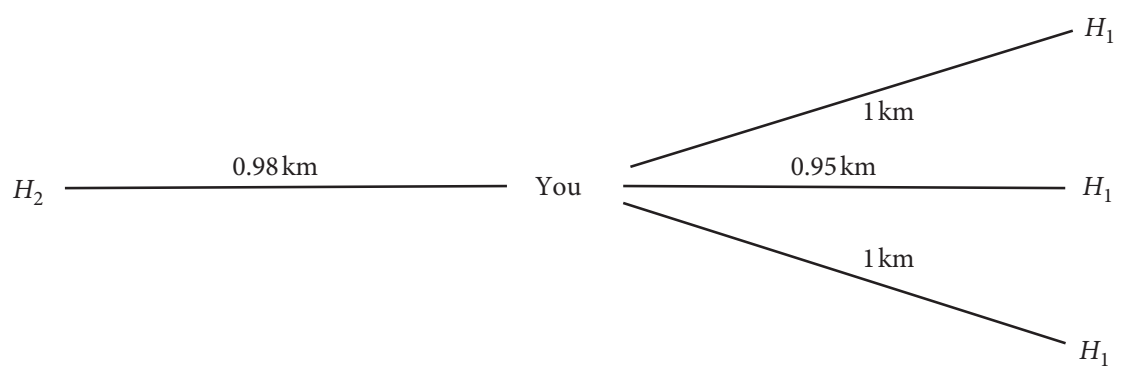

FIgURE 2: A decision-making example.

(4) $a^{\lambda_{1}+\lambda_{2}}=a^{\lambda_{1}} \otimes a^{\lambda_{2}}$

(5) $\operatorname{Ref}(\operatorname{Ref}(a))=a$

(6) $\operatorname{Rot}_{\theta_{1}}\left(\operatorname{Rot}_{\theta_{2}}(a)\right)=\operatorname{Rot}_{\theta_{1}+\theta_{2}}(a)$

(7) $\operatorname{Ref}\left(a^{\lambda_{1}}\right)=\operatorname{Ref}(a)^{\lambda_{1}}$

(8) $\operatorname{Ref}(a \otimes b)=\operatorname{Ref}(a) \otimes \operatorname{Ref}(b)$

Proof. Let $a=\left[\underline{r_{a}}, \overline{r_{a}}\right] \cdot e^{j v_{a}}, b=\left[\underline{r_{b}}, \overline{r_{b}}\right] \cdot e^{j v_{b}}$, and $c=$ $\left[r_{c}, \overline{r_{c}}\right] \cdot e^{j v_{c}}$.

(1) By the definition of multiplication of two IVCFVs, we have

$$
\begin{aligned}
a \otimes b & =\left[\underline{r_{a}} \cdot \underline{r_{b}}, \overline{r_{a}} \cdot \overline{r_{b}}\right] \cdot e^{j\left(v_{a}+v_{b}\right)} \\
& =\left[\underline{r_{b}} \cdot \underline{r_{a}}, \overline{r_{b}} \cdot \overline{r_{a}}\right] \cdot e^{j\left(v_{b}+v_{a}\right)} \\
& =b \otimes a .
\end{aligned}
$$

(2) And

$$
\begin{aligned}
(a \otimes b) \otimes c & =\left[\underline{r_{a}} \cdot \underline{r_{b}}, \overline{r_{a}} \cdot \overline{r_{b}}\right] \cdot e^{j\left(v_{a}+v_{b}\right)} \otimes\left[\underline{r_{c}}, \overline{r_{c}}\right] \cdot e^{j v_{c}} \\
& =\left[\underline{r_{a}}, \overline{r_{a}}\right] \cdot e^{j v_{a}} \otimes\left[\underline{r_{b}} \cdot \underline{r_{c}}, \overline{r_{b}} \cdot \overline{r_{c}}\right] \cdot e^{j\left(v_{b}+v_{c}\right)} \\
& =a \otimes(b \otimes c) .
\end{aligned}
$$

(3) By the definition of power of two IVCFVs, we have

$$
\begin{aligned}
(a \otimes b)^{\lambda_{1}} & =\left[\left[\underline{r_{a}} \cdot \underline{r_{b}}, \overline{r_{a}} \cdot \overline{r_{b}}\right] \cdot e^{j\left(v_{a}+v_{b}\right)}\right]^{\lambda_{1}} \\
& =\left[\left(\underline{r_{a}} \cdot \underline{r_{b}}\right)^{\lambda_{1}},\left(\overline{r_{a}} \cdot \overline{r_{b}}\right)^{\lambda_{1}}\right] \cdot e^{j \lambda_{1}\left(v_{a}+v_{b}\right)} \\
& =\left[\underline{r_{a}^{\lambda_{1}}} \cdot \underline{r_{b}}{ }^{\lambda_{1}}, \overline{r_{a}} \lambda_{1} \cdot \bar{r}_{b}^{\lambda_{1}}\right] \cdot e^{j\left(\lambda_{1} v_{a}+\lambda_{1} v_{b}\right)} \\
& =a^{\lambda_{1}} \otimes b^{\lambda_{1}} .
\end{aligned}
$$

(4) And

$$
\begin{aligned}
a^{\lambda_{1}+\lambda_{2}} & =\left[\underline{r_{a}^{\lambda_{1}+\lambda_{2}}}, \bar{r}_{a}^{\lambda_{1}+\lambda_{2}}\right] \cdot e^{j\left(\lambda_{1}+\lambda_{2}\right) v_{a}} \\
& =\left[\underline{r_{a}}{ }^{\lambda_{1}} \cdot \underline{r_{a}}, \overline{r_{a}} \lambda_{1} \cdot \bar{r}_{a}^{\lambda_{2}}\right] \cdot e^{j\left(\lambda_{1} v_{a}+\lambda_{2} v_{a}\right)} \\
& =a^{\lambda_{1}+\lambda_{2}} .
\end{aligned}
$$

(5) By the definition of reflection of two IVCFVs, we have

$$
\begin{aligned}
\operatorname{Ref}(\operatorname{Ref}(a)) & =\operatorname{Ref}\left(\left[\underline{r_{a}}, \overline{r_{a}}\right] \cdot e^{-j v_{a}}\right) \\
& =\left[\underline{r_{a}}, \overline{r_{a}}\right] \cdot e^{-\left(-j v_{a}\right)} \\
& =\left[\underline{r_{a}}, \overline{r_{a}}\right] \cdot e^{j v_{a}} \\
& =a .
\end{aligned}
$$

(6) By the definition of rotation of two IVCFVs, we have

$$
\begin{aligned}
\operatorname{Rot}_{\theta_{1}}\left(\operatorname{Rot}_{\theta_{2}}(a)\right) & =\operatorname{Rot}_{\theta_{1}}\left(\left[\underline{r_{a}}, \overline{r_{a}}\right] \cdot e^{j\left(v_{a}+\theta_{2}\right)}\right) \\
& =\left[\underline{r_{a}}, \overline{r_{a}}\right] \cdot e^{j\left(v_{a}+\theta_{2}+\theta_{1}\right)} \\
& =\operatorname{Rot}_{\theta_{1}+\theta_{2}}(a) .
\end{aligned}
$$

(7) For any two IVCFVs $a, b$ and real value $\lambda>0$, we have

$$
\begin{aligned}
\operatorname{Ref}(a)^{\lambda} & =\left[\left[\underline{r_{a}}, \overline{r_{a}}\right] \cdot e^{-j v_{a}}\right]^{\lambda} \\
& =\left[\underline{r_{a}^{\lambda}}, \overline{r_{a}}\right] \cdot e^{j\left(-\lambda v_{a}\right)} \\
& =\operatorname{Ref}\left(a^{\lambda}\right) .
\end{aligned}
$$

(8) For any two IVCFVs $a, b$, we have

$$
\begin{aligned}
\operatorname{Ref}(a) \otimes \operatorname{Ref}(b) & =\left[\underline{r_{a}}, \overline{r_{a}}\right] \cdot e^{-j v_{a}} \otimes\left[\underline{r_{b}}, \overline{r_{b}}\right] \cdot e^{-j v_{b}} \\
& =\left[\underline{r_{a}} \cdot \underline{r_{b}}, \overline{r_{a}} \cdot \overline{r_{b}}\right] \cdot e^{j\left(-v_{a}-v_{b}\right)} \\
& =\left[\underline{r_{a}} \cdot \underline{r_{b}}, \overline{r_{a}} \cdot \overline{r_{b}}\right] \cdot e^{-j\left(v_{a}+v_{b}\right)} \\
& =\operatorname{Ref}(a \otimes b) .
\end{aligned}
$$

Moreover, the multiplication and power operators have the following properties.

Theorem 2. Suppose that $a=\left[r_{a}, \overline{r_{a}}\right] \cdot e^{j v_{a}}, b=\left[r_{b}, \overline{r_{b}}\right] \cdot e^{j v_{b}}$, $c=\left[r_{c}, \overline{r_{c}}\right] \cdot e^{j v_{c}}$, and $d=\left[r_{d}, \overline{r_{d}}\right] \cdot e^{j v_{d}}$ are four IVCFVs; the parameters are $\lambda_{1}>0, \lambda_{2}>0$, and $\theta \in \mathbb{R}$. Then we have

(1) (Amplitude monotonicity) If $|a| \leq|b|,|c| \leq|d|$, then $|a \otimes c| \leq|b \otimes d|$ 
(2) (Amplitude boundedness) $\left[\underline{r_{a}} \wedge \underline{r_{b}}, \overline{r_{a}} \wedge \overline{r_{b}}\right] \leq|a \otimes b| \leq 1$

(3) If $\lambda_{1} \leq \lambda_{2}$, then $\left|a^{\lambda_{2}}\right| \leq\left|a^{\lambda_{1}}\right|$

(4) If $|a| \leq|b|$, then $\left|a^{\lambda_{1}}\right| \leq\left|a^{\lambda_{1}}\right|,|\operatorname{Ref}(a)| \leq|\operatorname{Ref}(b)|$, $\left|\operatorname{Rot}_{\theta}(a)\right| \leq\left|\operatorname{Rot}_{\theta}(a)\right|$

Proof. Since the amplitude terms of IVCFVs are interval values, we can obtain the above results from the properties of multiplication operator of interval values.

Rotational invariance and reflectional invariance are two important geometric properties of complex fuzzy operators $[28,29,39]$. They show that a complex fuzzy operator is invariant under a rotation and a reflection, respectively. We define the following two similar properties for intervalvalued complex fuzzy operators as follows.

(i) An interval-valued complex fuzzy operator $v:\left(\mathscr{I}^{[0,1]} \cdot \dot{D}\right)^{n} \longrightarrow \mathscr{I}^{[0,1]} \cdot \dot{D}$ is rotationally invariant if and only if, for any $\theta$,

$v\left(\operatorname{Rot}_{\theta}\left(a_{1}\right), \ldots, \operatorname{Rot}_{\theta}\left(a_{n}\right)\right)=\operatorname{Rot}_{\theta}\left(v\left(a_{1}, \ldots, a_{n}\right)\right)$.

(ii) An interval-valued complex fuzzy operator $v:\left(\mathscr{I}^{[0,1]} \cdot \dot{D}\right)^{n} \longrightarrow \mathscr{I}^{[0,1]} \cdot \dot{D}$ is reflectionally invariant if and only if

$$
v\left(\operatorname{Ref}\left(a_{1}\right), \ldots, \operatorname{Ref}\left(a_{n}\right)\right)=\operatorname{Ref}\left(v\left(a_{1}, \ldots, a_{n}\right)\right) .
$$

Then, for the multiplication operator of IVCFVs, we have the following results.

Theorem 3. The multiplication operator of IVCFVs is reflectionally invariant.

Proof. Trivial from Theorem 1 (16).

Theorem 4. The multiplication operator of IVCFVs is not rotationally invariant.

Proof. For any two IVCFVs $a=\left[\underline{r_{a}}, \overline{r_{a}}\right] \cdot e^{j v_{a}}, b=\left[\underline{r_{b}}, \overline{r_{b}}\right]$. $e^{j \nu_{b}}$ and real value $\theta$, we have

$$
\begin{aligned}
& \operatorname{Rot}_{\theta}(a) \otimes \operatorname{Rot}_{\theta}(b) \\
& =\left[\underline{r_{a}}, \overline{r_{a}}\right] \cdot e^{j\left(v_{a}+\theta\right)} \otimes\left[\underline{r_{b}}, \overline{r_{b}}\right] \cdot e^{j\left(v_{b}+\theta\right)} \\
& =\left[\underline{r_{a}} \cdot \underline{r_{b}}, \overline{r_{a}} \cdot \overline{r_{b}}\right] \cdot e^{j\left(v_{a}+v_{b}+2 \theta\right)}, \\
\operatorname{Rot}_{\theta}(a \otimes b) & =\left[\underline{r_{a}} \cdot \underline{r_{b}}, \overline{r_{a}} \cdot \overline{r_{b}}\right] \cdot e^{j\left(v_{a}+v_{b}+\theta\right)} .
\end{aligned}
$$

Since $v_{a}+v_{b}+2 \theta \neq v_{a}+v_{b}+\theta$, the multiplication operator is not rotationally invariant.

\section{Interval-Valued Complex Fuzzy Weighted Geometric Operators}

In this section, we introduce the weighted geometric operators in an interval-valued complex fuzzy environment and discuss their fundamental characteristics.
Definition 1. Let $a_{i}(i=1,2, \ldots, n)$ be a collection of IVCFVs; an interval-valued complex fuzzy weighted geometric (IVCFWG) operator is defined as

$$
\operatorname{IVCFWG}\left(a_{1}, a_{2}, \ldots, a_{n}\right)=\otimes_{i=1}^{n} a_{i}^{w_{i}},
$$

where $w_{i} \in[0,1]$ for all $i$ and $\sum_{i=1}^{n} w_{i}=1$.

When $w_{i}=1 / n(i=1,2, \ldots, n)$, then the IVCFWG operator is denoted by interval-valued complex fuzzy geometric (IVCFA) operator, i.e.,

$$
\operatorname{IVCFWG}\left(a_{1}, a_{2}, \ldots, a_{n}\right)=\otimes_{i=1}^{n} a_{i}^{1 / n} .
$$

When $a_{i} \in \mathscr{I}^{[0,1]}(i=1,2, \ldots, n)$, the IVCFWG operator can reduce to a traditional interval-valued fuzzy weighted geometric operator of values on unit interval $\mathscr{F}^{[0,1]}$.

When $a_{i} \in[0,1](i=1,2, \ldots, n)$, the IVCFWG operator can reduce to a traditional weighted geometric operator [49] of real numbers on unit interval $[0,1]$.

When $a_{i} \in[0,1]$ and $w_{i}=1 / n(i=1,2, \ldots, n)$, the CFWP operator can reduce to a traditional geometric mean operator [50] of real numbers on unit interval $[0,1]$.

Theorem 5. Let $a_{i}(i=1,2, \ldots, n)$ be a collection of IVCFVs; then, the aggregated value IVCFWG $\left(a_{1}, a_{2}, \ldots, a_{n}\right)$ is also an $I V C F V$ and

$$
\begin{aligned}
& \operatorname{IVCFWG}\left(a_{1}, a_{2}, \ldots, a_{n}\right) \\
& =\left[\prod_{i=1}^{n} \frac{w_{i}}{r_{a_{i}}}, \prod_{i=1}^{n} \overline{r_{a_{i}}} w_{i}\right] \cdot e^{j\left(\sum_{i=1}^{n} w_{i} v_{a_{i}}\right),}
\end{aligned}
$$

where $w_{i} \in[0,1]$ for all $i$ and $\sum_{i=1}^{n} w_{i}=1$.

Proof. It is easy to check that the aggregated value is also an IVCFV. Now, we prove equation (20) by mathematical induction method.

For $n=2$, we have $a_{1}^{w_{1}}=\left[r_{a_{1}} w_{1}, \bar{r}_{a_{1}}{ }^{w_{1}}\right] \cdot e^{j w_{1} v_{a_{1}}}$, $a_{2}^{w_{2}}=\left[r_{a_{2}}{ }^{w_{2}}, \overline{r_{a_{2}}} w_{2}\right] \cdot e^{j w_{2} v_{a_{2}}}$; then,

$$
\begin{aligned}
& \operatorname{IVCFWG}\left(a_{1}, a_{2}\right) \\
& =a_{1}^{w_{1}} \otimes a_{2}^{w_{2}} \\
& =\left[\underline{r_{a_{1}}} \underline{w_{a_{2}}}, \bar{r}_{a_{1}}^{w_{1}} \bar{r}_{a_{2}}^{w_{2}}\right] \cdot e^{j\left(w_{1} v_{a_{1}}+w_{2} v_{a_{2}}\right)} \\
& =\left[\prod_{i=1}^{2} \frac{r_{a_{i}}}{w_{1}}, \prod_{i=1}^{2} \overline{r_{a_{i}}} w_{i}\right] \cdot e^{j\left(\sum_{i=1}^{2} w_{i} v_{a_{i}}\right)} .
\end{aligned}
$$

If equation (20) holds for $n=k$, i.e.,

$$
\begin{aligned}
& \operatorname{IVCFWG}\left(a_{1}, a_{2}, \ldots, a_{k}\right) \\
& =\left[\prod_{i=1}^{k} r_{a_{i}}^{w_{1}}, \prod_{i=1}^{k} \overline{r_{a_{i}}} w_{i}\right] \cdot e^{j\left(\sum_{i=1}^{k} w_{i} v_{a_{i}}\right)},
\end{aligned}
$$

then for $n=k+1$, 


$$
\begin{aligned}
& \operatorname{IVCFWG}\left(a_{1}, a_{2}, \ldots, a_{k+1}\right) \\
& =\operatorname{IVCFWG}\left(a_{1}, a_{2}, \ldots, a_{k}\right) \otimes a_{k+1}^{w_{k+1}} \\
& =\left[\prod_{i=1}^{k} \frac{w_{a_{i}}}{w_{i}}, \prod_{i=1}^{k} \overline{r_{a_{i}}} w_{i}\right] \cdot e^{j\left(\sum_{i=1}^{k} w_{i} v_{a_{i}}\right) \otimes a_{k+1}^{w_{k+1}}} \\
& =\left[\left(\prod_{i=1}^{k} \frac{w_{a_{i}}}{r}\right) \cdot \underline{r_{a_{k+1}}},\left(\prod_{i=1}^{k} \overline{r_{a_{i}}} w_{i}\right) \cdot \overline{r_{a_{k+1}}} w_{k+1}\right] \\
& \left.\cdot e^{j\left(\sum_{i=1}^{k} w_{i} v_{a_{i}}+w_{k+1} v_{a_{k+1}}\right.}\right) \\
& =\left[\prod_{i=1}^{k+1} \frac{w_{a_{i}}}{r_{i}} \prod_{i=1}^{k+1} \overline{r_{a_{i}}} w_{i}\right] \cdot e^{j\left(\sum_{i=1}^{k+1} w_{i} v_{a_{i}}\right)} .
\end{aligned}
$$

Therefore, equation (20) holds for all $n$.

The working of the proposed IVCFWG operator is explained with a numerical example as follows.

Example 1. Let $a_{1}=[0.6,0.7] \cdot e^{j 0.1 \pi}, a_{2}=[0.3,0.5] \cdot e^{j 0.2 \pi}$, $a_{3}=[0.6,0.8] \cdot e^{j 0.7 \pi}$, and $a_{4}=[0.4,0.7] \cdot e^{j 0.4 \pi}$ be four IVCFVs and $w=(0.1,0.2,0.3,0.4)^{T}$ be the associated weight vector. Then, by using equation (20),

$$
\begin{aligned}
\prod_{i=1}^{4} \underline{r_{a_{i}}} & =0.6^{0.1} \cdot 0.3^{0.2} \cdot 0.6^{0.3} \cdot 0.4^{0.4}=0.4441, \\
\prod_{i=1}^{4} \bar{r}_{a_{i}} w_{i} & =0.7^{0.1} \cdot 0.5^{0.2} \cdot 0.8^{0.3} \cdot 0.7^{0.4}=0.6812, \\
\sum_{i=1}^{4} w_{i} \nu_{a_{i}} & =0.1 \cdot 0.1 \pi+0.2 \cdot 0.2 \pi+0.3 \cdot 0.7 \pi+0.4 \cdot 0.4 \pi \\
& =0.42 \pi,
\end{aligned}
$$

we obtain $\operatorname{IVCFWG}\left(a_{1}, a_{2}, a_{3}, a_{4}\right)=[0.4441,0.6812]$. $e^{j 0.42 \pi}$.

Based on Theorem 5, the proposed IVCFWG operator satisfies the following properties.

Theorem 6. Let $a_{i}(i=1,2, \ldots, n)$ and $b_{i}(i=1,2, \ldots, n)$ be two collections of IVCFVs, the weights be $w_{i} \in[0,1](i=$ $1,2, \ldots, n)$, and $\sum_{i=1}^{n} w_{i}=1$. Then, we have the following properties:

(1) (Idempotency) If $a_{i}=a(i=1,2, \ldots, n)$, then

$$
\operatorname{IVCFWG}\left(a_{1}, a_{2}, \ldots, a_{n}\right)=a \text {. }
$$

(2) (Amplitude monotonicity) If $\left|a_{i}\right| \leq\left|b_{i}\right|(i=1,2, \ldots, n)$, then

$$
\begin{aligned}
& \left|\operatorname{IVCFWG}\left(a_{1}, a_{2}, \ldots, a_{n}\right)\right| \\
\leq & \left|\operatorname{IVCFWG}\left(b_{1}, b_{2}, \ldots, b_{n}\right)\right| .
\end{aligned}
$$

(3) (Amplitude boundedness)

$$
r_{1} \leq\left|\operatorname{IVCFWG}\left(a_{1}, a_{2}, \ldots, a_{n}\right)\right| \leq r_{2},
$$

where $r_{1}, r_{2}$ are two interval values:

$$
\begin{aligned}
& r_{1}=\left[\min _{i} \underline{r_{a_{i}}}, \min _{i} \overline{r_{a_{i}}}\right], \\
& r_{2}=\left[\max _{i} \underline{r_{a_{i}}}, \max _{i} \overline{r_{a_{i}}}\right] .
\end{aligned}
$$

Proof.

(1) Let $a_{i}=a=\left[\underline{r_{a}}, \overline{r_{a}}\right],(i=1,2, \ldots, n)$; then,

$$
\begin{aligned}
& \operatorname{IVCFWG}\left(a_{1}, a_{2}, \ldots, a_{n}\right) \\
& =\left[\prod_{i=1}^{n} \underline{r_{a}^{w_{i}}}, \prod_{i=1}^{n} \overline{r_{a}} w_{i}\right] \cdot e^{j\left(\sum_{i=1}^{n} w_{i} v_{a}\right)} \\
& =\left[\underline{r_{a}} \sum_{i=1}^{n} w_{i}, \overline{r_{a}} \sum_{i=1}^{n} w_{i}\right] \cdot e^{j v_{a}} \\
& =\left[\underline{r_{a}}, \overline{r_{a}}\right] \cdot e^{j v_{a}} \\
& =a .
\end{aligned}
$$

(2) The property of the amplitude monotonicity is trivial from Theorem 2 (4).

(3) The property of the amplitude boundedness is easily obtained from idempotency and amplitude monotonicity.

Note that idempotency is concerned with both the phase part and amplitude part of IVCFVs. Amplitude boundedness and amplitude monotonicity are only concerned with the amplitude part of IVCFVs.

Theorem 7. The IVCFWG operator is reflectionally invariant.

Proof. For any collection of IVCFVs $a_{i}(i=1,2, \ldots, n)$, from equation (20), we have

$$
\begin{aligned}
& \operatorname{IVCFWG}\left(\operatorname{Ref}\left(a_{1}\right), \operatorname{Ref}\left(a_{2}\right), \ldots, \operatorname{Ref}\left(a_{n}\right)\right) \\
& =\left[\prod_{i=1}^{n}\left(\underline{r_{a_{i}}}\right)^{w_{i}}, \prod_{i=1}^{n}\left(\overline{r_{a_{i}}}\right)^{w_{i}}\right] \cdot e^{j\left(\sum_{i=1}^{n}-w_{i} v_{a_{i}}\right)} \\
& =\left[\prod_{i=1}^{n}\left(\underline{r_{a_{i}}}\right)^{w_{i}}, \prod_{i=1}^{n}\left(\overline{r_{a_{i}}}\right)^{w_{i}}\right] \cdot e^{-j\left(\sum_{i=1}^{n} w_{i} v_{a_{i}}\right)}, \\
& \operatorname{Ref}\left(\operatorname{IVCFWG}\left(a_{1}, a_{2}, \ldots, a_{n}\right)\right) \\
& =\operatorname{Ref}\left(\otimes_{i=1}^{n} a_{i}^{w_{i}}\right) \\
& \left.=\left[\prod_{i=1}^{n}\left(\underline{r_{a_{i}}}\right)^{w_{i}}, \prod_{i=1}^{n}\left(\overline{r_{a_{i}}}\right)^{w_{i}}\right] \cdot e^{-j\left(\sum_{i=1}^{n} w_{i} v_{a_{i}}\right.}\right) .
\end{aligned}
$$


Then, $\quad$ IVCFWG $\left(\operatorname{Ref}\left(a_{1}\right), \operatorname{Ref}\left(a_{2}\right), \ldots, \operatorname{Ref}\left(a_{n}\right)\right)=$ $\operatorname{Ref}\left(\otimes_{i=1}^{n} w_{i} a_{i}\right)$ is reflectionally invariant.

Theorem 8. The IVCFWG operator is rotationally invariant.

Proof. For any collection of IVCFVs $a_{i}(i=1,2, \ldots, n)$ and any $\theta \in \mathbb{R}$, we have

$$
\begin{aligned}
& \operatorname{IVCFWG}\left(\operatorname{Rot}_{\theta}\left(a_{1}\right), \operatorname{Rot}_{\theta}\left(a_{2}\right), \ldots, \operatorname{Rot}_{\theta}\left(a_{n}\right)\right) \\
& =\left[\prod_{i=1}^{n}\left(\underline{r_{a_{i}}}\right)^{w_{i}}, \prod_{i=1}^{n}\left(\overline{r_{a_{i}}}\right)^{w_{i}}\right] \cdot e^{j\left(\sum_{i=1}^{n} w_{i}\left(v_{a_{i}}+\theta\right)\right)} \\
& =\left[\prod_{i=1}^{n}\left(\underline{r_{a_{i}}}\right)^{w_{i}}, \prod_{i=1}^{n}\left(\overline{r_{a_{i}}}\right)^{w_{i}}\right] \cdot e^{j\left(\left(\sum_{i=1}^{n} w_{i} v_{a_{i}}\right)+\left(\sum_{i=1}^{n} w_{i} \theta\right)\right)} \\
& =\left[\prod_{i=1}^{n}\left(\underline{r_{a_{i}}}\right)^{w_{i}}, \prod_{i=1}^{n}\left(\overline{r_{a_{i}}}\right)^{w_{i}}\right] \cdot e^{j\left(\left(\sum_{i=1}^{n} w_{i} v_{a_{i}}\right)+\theta\right)} \\
& ={ }_{\operatorname{Rot} \theta}\left(\operatorname{IVCFWG}\left(a_{1}, a_{2}, \ldots, a_{n}\right)\right) .
\end{aligned}
$$

Then, the IVCFWG operator is rotationally invariant.

\section{Interval-Valued Complex Fuzzy Ordered Weighted Geometric Operators}

Based on the order of IVCFVs defined by equation (2) and the ordered weighted averaging (OWA) operator introduced by Yager [1], we define an interval-valued complex fuzzy ordered weighted geometric (IVCFOWG) operator as follows.

Definition 2. Let $a_{i}(i=1,2, \ldots, n)$ be a collection of CFVs; then, an IVCFOWG operator is defined as

$$
\operatorname{IVCFOWG}\left(a_{1}, a_{2}, \ldots, a_{n}\right)=\otimes_{i=1}^{n} a_{\sigma(i)}^{w_{i}},
$$

where $\quad w_{i} \in[0,1](i=1,2, \ldots, n) \quad$ and $\quad \sum_{i=1}^{n} w_{i}=1$, $(\sigma(1), \sigma(2), \ldots, \sigma(n))$ is a permutation of $(1,2, \ldots, n)$ such that $a_{\sigma(i-1)} \geq a_{\sigma(i)}$ for all $i$.

Especially, when $w_{i}=1 / n(i=1,2, \ldots, n)$, the IVCFOWG operator is reduced to the IVCFWG operator.

Similar to the IVCFWG operator, the IVCFOWG operator has the following properties.

Theorem 9. Let $a_{i}(i=1,2, \ldots, n)$ be a collection of IVCFVs; then, the aggregated value IVCFOWG $\left(a_{1}, a_{2}, \ldots, a_{n}\right)$ is also an IVCFV and

$$
\begin{aligned}
& \operatorname{IVCFOWG}\left(a_{1}, a_{2}, \ldots, a_{n}\right) \\
& =\left[\prod_{i=1}^{n}\left(\underline{r_{a_{(\sigma i)}}}\right)^{w_{i}}, \prod_{i=1}^{n}\left(\overline{r_{a_{(\sigma i)}}}\right)^{w_{i}}\right] \cdot e^{j\left(\sum_{i=1}^{i=1} w_{i} a_{\sigma(i)}\right)},
\end{aligned}
$$

where $w_{i} \in[0,1]$ for all $i$ and $\sum_{i=1}^{n} w_{i}=1$, $(\sigma(1), \sigma(2), \ldots, \sigma(n))$ is a permutation of $(1,2, \ldots, n)$ such that $a_{\sigma(i-1)} \geq a_{\sigma(i)}$ for all $i$.
Proof. The proof is similar to Theorem 5.

The working of the proposed IVCFOWG operator is explained with a numerical example as follows.

Example 2. Let $a_{1}=[0.6,0.7] \cdot e^{j 0.1 \pi}, a_{2}=[0.3,0.5] \cdot e^{j 0.2 \pi}$, $a_{3}=[0.6,0.8] \cdot e^{j 0.7 \pi}$, and $a_{4}=[0.4,0.7] \cdot e^{j 0.4 \pi}$ be four IVCFVs and $w=(0.1,0.2,0.3,0.4)^{T}$ be the associated weight vector. By the order from equation (2), we have $a_{3} \geq a_{1} \geq a_{4} \geq a_{2}$. Then, by using equation (33),

$$
\begin{aligned}
\prod_{i=1}^{4} \frac{r_{a_{\sigma(i)}}^{w_{i}}}{} & =0.6^{0.1} \cdot 0.6^{0.2} \cdot 0.4^{0.3} \cdot 0.3^{0.4}=0.4026 \\
\prod_{i=1}^{4} \frac{w_{i}}{r_{a_{\sigma(i)}}} & =0.8^{0.1} \cdot 0.8^{0.2} \cdot 0.7^{0.3} \cdot 0.5^{0.4}=0.6369 \\
\sum_{i=1}^{4} w_{i} v_{a_{i}} & =0.1 \cdot 0.7 \pi+0.2 \cdot 0.1 \pi+0.3 \cdot 0.4 \pi+0.4 \cdot 0.2 \pi \\
& =0.29 \pi
\end{aligned}
$$

we obtain IVCFOWG $\left(a_{1}, a_{2}, a_{3}, a_{4}\right)=[0.4026,0.6369]$. $e^{j 0.29 \pi}$. It is different from $\operatorname{IVCFWG}\left(a_{1}, a_{2}, a_{3}, a_{4}\right)=$ $[0.4441,0.6812] \cdot e^{j 0.42 \pi}$ (see Example 1).

Theorem 10. Let $a_{i}(i=1,2, \ldots, n)$ be a collection of CFVs, CFOWP weights be $w_{i} \in[0,1](i=1,2, \ldots, n)$, and $\sum_{i=1}^{n} w_{i}=1$. Then, we have the following properties.

(1) (Idempotency) If $a_{i}=a(i=1,2, \ldots, n)$, then

$$
\operatorname{IVCFOWG}\left(a_{1}, a_{2}, \ldots, a_{n}\right)=a \text {. }
$$

(2) (Amplitude monotonicity) If $\left|a_{i}\right| \leq\left|b_{i}\right|(i=1,2, \ldots$, $n)$, then

$$
\begin{aligned}
& \left|\operatorname{IVCFWG}\left(a_{1}, a_{2}, \ldots, a_{n}\right)\right| \\
\leq & \left|\operatorname{IVCFWG}\left(b_{1}, b_{2}, \ldots, b_{n}\right)\right| .
\end{aligned}
$$

(3) (Amplitude boundedness)

$$
r_{1} \leq\left|\operatorname{IVCFOWG}\left(a_{1}, a_{2}, \ldots, a_{n}\right)\right| \leq r_{2} .
$$

where $r_{1}, r_{2}$ are two interval values:

$$
\begin{aligned}
& r_{1}=\left[\min _{i} \underline{r_{a_{i}}}, \min _{i} \overline{r_{a_{i}}}\right], \\
& r_{2}=\left[\max _{i} \underline{r_{a_{i}}}, \max _{i} \overline{r_{a_{i}}}\right] .
\end{aligned}
$$

Proof. The proof is similar to Theorem 6.

Theorem 11. The IVCFOWG operator is reflectionally invariant and rotationally invariant.

Proof. The proof is similar to Theorems 7 and 8 . 
Note that the operator $\otimes$ does not have the property of rotational invariance, but the IVCFWG and IVCFOWG operators defined based on $\otimes$ operator have the property of rotational invariance.

Here, we investigate the aggregation operators based on special class of the IVCFVs, which belong to subsets of the upper-right quadrant of the complex unit disk. Let $D_{1}=\left\{e^{j v} \mid v \in[0, \pi / 2]\right\}$; we consider the aggregation operator on $\mathscr{I}^{[0,1]} \cdot D_{1}$.

Let us consider the closeness of IVCFVs on $\mathscr{I}^{[0,1]} \cdot D_{1}$ under the IVCFWG and IVCFOWG operations. For the IVCFWG operator, we have the following result.

Theorem 12. Let $z_{1}, z_{2}, \ldots, z_{n} \in \mathscr{I}^{[0,1]} \cdot D_{1}$. Then, the aggregated value

$$
\operatorname{IVCFWG}\left(z_{1}, z_{2}, \ldots, z_{n}\right) \in \mathscr{I}^{[0,1]} \cdot D_{1} \text {. }
$$

Proof. Denoting IVCFWG $\left(z_{1}, z_{2}, \ldots, z_{n}\right)=[\underline{t}, \bar{t}] \cdot e^{j v}$, from Theorem 5, $[\underline{t}, \bar{t}]$ is an interval value. Since $v=\sum_{i=1}^{n} w_{i} \cdot v_{z_{i}}$ is a weighted arithmetic aggregation operator of real numbers on $[0, \pi / 2]$, then we have $v \in[0, \pi / 2]$. Thus, $\operatorname{IVCFWG}\left(z_{1}, z_{2}, \ldots, z_{n}\right) \in \mathscr{I}^{[0,1]} \cdot D_{1}$.

Similar to the above theorem, we have the following.

Theorem 13. Let $z_{1}, z_{2}, \ldots, z_{n} \in \mathscr{I}^{[0,1]} \cdot D_{1}$. Then, the aggregated value

$$
\operatorname{IVCFOWG}\left(z_{1}, z_{2}, \ldots, z_{n}\right) \in \mathscr{I}^{[0,1]} \cdot D_{1} .
$$

The above theorems show us that the IVCFWG and the IVCFOWG operators are close under values on $\mathscr{I}^{[0,1]} \cdot D_{1}$.

Consider other quadrants of the complex unit disk. Let

$$
D_{k}=\left\{z=e^{j \theta} \mid \theta \in\left[\frac{(k-1) \pi}{2}, \frac{k \pi}{2}\right]\right\},
$$

for $k=1$ to 4 .

Now, we discuss the closeness of the IVCFWG and the IVCFOWG operators on values of other quadrants of the complex unit disk. Plainly, we have the following.

Theorem 14. For any $k \in\{1,2,3,4\}$, if $z_{1}, z_{2}, \ldots$, $z_{n} \in \mathscr{I}^{[0,1]} \cdot D_{k}$, then we have

$$
\begin{array}{r}
\operatorname{IVCFWG}\left(z_{1}, z_{2}, \ldots, z_{n}\right) \in \mathscr{I}^{[0,1]} \cdot D_{k}, \\
\operatorname{IVCFOWG}\left(z_{1}, z_{2}, \ldots, z_{n}\right) \in \mathscr{I}^{[0,1]} \cdot D_{k} .
\end{array}
$$

Proof. Similar to Theorem 12.

\section{An Approach to Decision Making with the IVCFWG Operator}

In this section, we present an approach using the IVCFWG operator to a decision making with interval-valued complex fuzzy information.

We consider a target selection application of CFSs. Assume that our position is fixed, and then we can measure the distance and angle of the possible alternatives by using a position sensor and an angular sensor (or other attributes from expert opinions). Assume that we get a measurement $(d \pm \epsilon, q)$. Here, we use an interval value $[\underline{d}, \bar{d}]$ to represent $d \pm \epsilon$ by setting $\underline{d}=d-\epsilon$ and $\bar{d}=d+\epsilon$. To improve the target location accuracy, we repeatedly measure the alternatives. Then, the target is selected in the following approach according to aggregation theory.

Let $x_{i}(i=1,2, \ldots, n)$ be the possible alternatives and $e_{k}(k=1,2, \ldots, m)$ be the experts. Then, the decision maker provides a decision matrix $A=\left(a_{i k}\right)_{n \times m}$, where $a_{i k}$ is an interval-valued complex fuzzy value given by the expert $e_{k}$ for alternative $x_{i}$. Further, assume that $w=\left(w_{1}, w_{2}, \ldots\right.$, $\left.w_{n}\right)^{T}$ is the weight vector of the different experts such that $w_{i} \geq 0(i=1,2, \ldots, n)$ and $\sum_{i=1}^{n} w_{i}=1$. The process can be summarized as follows:

Step 1. Transform the decision matrix $A$ into the normalized interval-valued complex fuzzy decision matrix $C=\left(c_{i k}\right)_{n \times m}$ using $c_{i k}=a_{i k} / d$ where $d=\max _{i k}$ $\left|a_{i k}\right|$.

Step 2. Aggregate all the interval-valued complex fuzzy values $a_{i k}(k=1,2, \ldots, m)$ and get the overall intervalvalued complex fuzzy value $b_{i}$ corresponding to the alternative $x_{i}$ by the IVCFWG operator, $b_{i}=$ IVCFWG $\left(a_{i 1}, a_{i 2}, \ldots, a_{i n}\right)$.

Step 3. Rank the overall IVCFVs $b_{i}(i=1,2, \ldots, n)$ using (2).

Next, we give an example to illustrate the above approach.

Example 3. Suppose we want to go to the nearest bank, then we often use GPS navigation system or ask strangers for directions.

Suppose that there are three alternatives $x_{i}(i=1,2,3)$ and three experts (GPS or stranger) $e_{i}(i=1,2,3)$ with the weight vector $(0.4,0.3,0.3)^{T}$. The three experts evaluate the three alternatives under the IVCFS environment, and their corresponding rating values are summarized in the decision matrix $C=\left(a_{i k}\right)_{3 \times 3}$ represented in Table 1 , where $c_{i k}=r_{i k}$. $e^{j v_{i k} \pi}$ is an interval-valued complex fuzzy value, $\left[r_{i k}, \overline{r_{i k}}\right]$ represents the distance between $r_{i k} \mathrm{~km}$ and $\overline{r_{i k}} \mathrm{~km}, \overline{\text { and }} \nu_{i k}$ represents the direction for the alternative $x_{i}$.

Step 1. The values $c_{i k}$ do not need normalization.

Step 2. Aggregate the interval-valued complex fuzzy values $b_{i}$ of the alternatives $x_{i}$ by using the IVCFWG operator (see equation (20)):

$$
\begin{aligned}
\prod_{i=1}^{3} \frac{r_{a_{1 i}}^{w_{i}}}{} & =0.19^{0.4} \cdot 0.11^{0.3} \cdot 0.16^{0.3}=0.1532, \\
\prod_{i=1}^{3} \frac{w_{a_{1 i}}}{w_{i}} & =0.25^{0.4} \cdot 0.23^{0.3} \cdot 0.23^{0.3}=0.2378, \\
\sum_{i=1}^{3} w_{i} v_{a_{1 i}} & =(0.4 \cdot 0.11+0.3 \cdot 0.13+0.3 \cdot 0.11) \cdot \pi \\
& =0.116 \pi,
\end{aligned}
$$


TABLe 1: Decision matrix.

\begin{tabular}{cccc}
\hline & $e_{1}$ & $e_{2}$ & $e_{3}$ \\
\hline$x_{1}$ & {$[0.19,0.25] \cdot e^{j 0.11 \pi}$} & {$[0.11,0.23] \cdot e^{j 0.13 \pi}$} & {$[0.16,0.23] \cdot e^{j 0.11 \pi}$} \\
$x_{2}$ & {$[0.12,0.23] \cdot e^{j 0.52 \pi}$} & {$[0.18,0.24] \cdot e^{j 0.54 \pi}$} & {$[0.18,0.20] \cdot e^{j 0.52 \pi}$} \\
$x_{3}$ & {$[0.10,0.16] \cdot e^{j 1.11 \pi}$} & {$[0.19,0.23] \cdot e^{j 1.12 \pi}$} & {$[0.17,0.19] \cdot e^{j 1.14 \pi}$} \\
\hline
\end{tabular}

and we obtain $b_{1}=\operatorname{IVCFWG}\left(a_{11}, a_{12}, a_{13}\right)=$ $[0.1532,0.2378] \cdot e^{j 0.116 \pi}$.

$$
\begin{aligned}
\prod_{i=1}^{3} \frac{r_{a_{2 i}}^{w_{i}}}{} & =0.12^{0.4} \cdot 0.18^{0.3} \cdot 0.18^{0.3}=0.1531, \\
\prod_{i=1}^{3} \frac{{ }_{r_{2 i}}}{w_{i}} & =0.23^{0.4} \cdot 0.24^{0.3} \cdot 0.2^{0.3}=0.2234, \\
\sum_{i=1}^{3} w_{i} \nu_{a_{2 i}} & =(0.4 \cdot 0.52+0.3 \cdot 0.54+0.3 \cdot 0.52) \cdot \pi \\
& =0.526 \pi,
\end{aligned}
$$

and we obtain $b_{2}=\operatorname{IVCFWG}\left(a_{11}, a_{12}, a_{13}\right)=[0.1531$, $0.2234] \cdot e^{j 0.126 \pi}$.

$$
\begin{aligned}
\prod_{i=1}^{3} \underline{r_{a_{3 i}}^{w_{i}}} & =0.1^{0.4} \cdot 0.19^{0.3} \cdot 0.17^{0.3}=0.1422, \\
\prod_{i=1}^{3} \frac{{ }_{r_{3 i}}}{w_{i}} & =0.16^{0.4} \cdot 0.23^{0.3} \cdot 0.19^{0.3}=0.1878, \\
\sum_{i=1}^{3} w_{i} \nu_{a_{3 i}} & =(0.4 \cdot 1.11+0.3 \cdot 1.12+0.3 \cdot 1.14) \cdot \pi \\
& =1.122 \pi,
\end{aligned}
$$

and we obtain $b_{3}=\operatorname{IVCFWG}\left(a_{11}, a_{12}, a_{13}\right)=[0.1422$, $0.1878] \cdot e^{j 0.122 \pi}$.

Step 3. Rank the interval-valued complex fuzzy values $b_{i}(i=1,2,3):\left|b_{1}\right|>\left|b_{2}\right|>\left|b_{3}\right|$, which shows that the alternative $x_{3}$ is the optimal choice, i.e., the nearest bank.

An IVCFS is a generalization of complex fuzzy sers (CFS), interval-valued fuzzy sets (IVFS), and fuzzy sets (FS). IVCFS can handle two-dimensional information in a single set. Then, IVCFS contains much more information than CFS, IVFS, and FS.

It is revealed from the present study that the aggregation operators under IVFSs and FSs $[5,6]$ are the special cases of the proposed aggregation operators. Thus, the proposed aggregation operators can be equivalently utilized to solve the DM problem under these existing environments by setting phase term to be zero while the existing operators $[5,6]$ are unable to solve the problems under the IVCFSs environment considered in the present paper. Thus, intervalvalued complex fuzzy aggregation operators are more general than some (interval-valued) fuzzy aggregation operators.

\section{Conclusion}

As mentioned in [50], the basic feature of aggregation operators is their monotonicity property. However, aggregation operators under the complex fuzzy environment [40] are not monotone. Interestingly, such aggregation operators can explain some phenomena in our real life.

In this paper, we discussed the geometric aggregation operators under the interval-valued complex fuzzy environment. Two interval-valued complex fuzzy aggregation operators, the IVCFWG and the IVCFOWG operators, are developed and their properties are studied. It is also interesting to note that both the IVCFWG and the IVCFOWG operators are rotationally invariant and reflectionally invariant. Further, based on the proposed operators, we presented a decision-making approach under interval-valued complex fuzzy information. An illustrative example is given for illustrating the proposed approach.

It was observed that the aggregation operator based on nonadditive integrals (Choquet [51] and Sugeno integrals [52]) is one of the hot topics in this field [49]. As future work, we can consider the complex fuzzy aggregation operators based on complex integrals.

\section{Data Availability}

The data used to support the findings of this study are included in the article.

\section{Conflicts of Interest}

The authors declare that they have no conflicts of interest.

\section{Acknowledgments}

This research was funded by the Open Foundation of Guangxi Colleges and Universities Key Laboratory of Complex System Optimization and Big Data Processing (grant no. 2017CSOBDP0103).

\section{References}

[1] R. R. Yager, "On ordered weighted averaging aggregation operators in multicriteria decisionmaking," IEEE Transactions on Systems, Man, and Cybernetics, vol. 18, no. 1, pp. 183-190, 1988.

[2] R. R. Yager, "The power average operator," IEEE Transactions on Systems, Man, and Cybernetics-Part A: Systems and Humans, vol. 31, no. 6, pp. 724-731, 2001.

[3] Z. S. Xu and R. R. Yager, "Power geometric operators and their use in group decision making," IEEE Transactions on Fuzzy Systems, vol. 18, pp. 94-105, 2010.

[4] L. Zhou, H. Chen, and J. Liu, "Generalized power aggregation operators and their applications in group decision making," Computers \& Industrial Engineering, vol. 62, no. 4, pp. 989999, 2012.

[5] Z. S. Xu, "Intuitionistic fuzzy aggregation operators," IEEE Transactions on Fuzzy Systems, vol. 15, no. 6, pp. 1179-1187, 2007. 
[6] Z. Xu and R. R. Yager, "Some geometric aggregation operators based on intuitionistic fuzzy sets," International Journal of General Systems, vol. 35, no. 4, pp. 417-433, 2006.

[7] Z. S. Xu, Intuitionistic Fuzzy Aggregation and Clustering, Springer, Berlin, Germany, 2013.

[8] Z. Xu, "Approaches to multiple attribute group decision making based on intuitionistic fuzzy power aggregation operators," Knowledge-Based Systems, vol. 24, no. 6, pp. 749760, 2011.

[9] P. Liu and Y. Wang, "Multiple attribute group decision making methods based on intuitionistic linguistic power generalized aggregation operators," Applied Soft Computing, vol. 17, pp. 90-104, 2014.

[10] P. Liu, "Some Hamacher aggregation operators based on the interval-valued intuitionistic fuzzy numbers and their application to group decision making," IEEE Transactions on Fuzzy Systems, vol. 22, no. 1, pp. 83-97, 2014.

[11] P. Liu, "Multiple attribute decision-making methods based on normal intuitionistic fuzzy interaction aggregation operators," Symmetry, vol. 9, no. 11, p. 261, 2017.

[12] D. Yu, Y. Wu, and T. Lu, "Interval-valued intuitionistic fuzzy prioritized operators and their application in group decision making," Knowledge-Based Systems, vol. 30, pp. 57-66, 2012.

[13] P. Liu, "Multiple attribute group decision making method based on interval-valued intuitionistic fuzzy power Heronian aggregation operators," Computers \& Industrial Engineering, vol. 108, pp. 199-212, 2017.

[14] P. Liu and H. Li, "Interval-valued intuitionistic fuzzy power Bonferroni aggregation operators and their application to group decision making," Cognitive Computation, vol. 9, no. 4, pp. 494-512, 2017.

[15] S. Zeng, Z. Mu, and T. Baležentis, "A novel aggregation method for Pythagorean fuzzy multiple attribute group decision making," International Journal of Intelligent Systems, vol. 33, no. 3, pp. 573-585, 2018.

[16] X. Zhang, "A novel approach based on similarity measure for Pythagorean fuzzy multiple criteria group decision making," International Journal of Intelligent Systems, vol. 31, no. 6, pp. 593-611, 2016.

[17] H. Garg, "A new generalized pythagorean fuzzy information aggregation using einstein operations and its application to decision making," International Journal of Intelligent Systems, vol. 31, no. 9, pp. 886-920, 2016.

[18] J. Ye, "A multicriteria decision-making method using aggregation operators for simplified neutrosophic sets," Journal of Intelligent \& Fuzzy Systems, vol. 26, no. 5, pp. 2459-2466, 2014.

[19] A. Tu, J. Ye, and B. Wang, "Multiple attribute decisionmaking method using similarity measures of neutrosophic cubic sets," Symmetry, vol. 10, no. 6, p. 215, 2018.

[20] A. Tu, J. Ye, and B. Wang, "Symmetry measures of simplified neutrosophic sets for multiple attribute decision-making problems," Symmetry, vol. 10, no. 5, p. 144, 2018.

[21] M. Xia and Z. Xu, "Hesitant fuzzy information aggregation in decision making," International Journal of Approximate Reasoning, vol. 52, no. 3, pp. 395-407, 2011.

[22] B. Zhu, Z. Xu, and M. Xia, "Hesitant fuzzy geometric Bonferroni means," Information Sciences, vol. 205, pp. 72-85, 2012.

[23] C. Song, Z. Xu, and H. Zhao, "A novel comparison of probabilistic hesitant fuzzy elements in multi-criteria decision making," Symmetry, vol. 10, no. 177, 2018.

[24] F. Amin, A. Fahmi, S. Abdullah, A. Ali, R. Ahmed, and F. Ghani, "Triangular cubic linguistic hesitant fuzzy aggregation operators and their application in group decision making," Journal of Intelligent and Fuzzy System, vol. 34, no. 4, pp. 2401-2416, 2018.

[25] A. Fahmi, S. Abdullah, F. Amin, A. Ali, and W. A. Khan, "Some geometric operators with triangular cubic linguistic hesitant fuzzy number and their application in group decision-making," Journal of Intelligent and Fuzzy Systems, vol. 35, no. 2, pp. 2485-2499, 2018.

[26] L. A. Zadeh, "Fuzzy sets," Information and Control, vol. 8, no. 3, pp. 338-353, 1965.

[27] D. Ramot, R. Milo, M. Friedman, and A. Kandel, "Complex fuzzy sets," IEEE Transactions on Fuzzy Systems, vol. 10, no. 2, pp. 171-186, 2002.

[28] S. Dick, "Toward complex fuzzy logic," IEEE Transactions on Fuzzy Systems, vol. 13, no. 3, pp. 405-414, 2005.

[29] S. Dai, "A generalization of rotational invariance for complex fuzzy operations," IEEE Transactions on Fuzzy Systems, p. 1, 2020.

[30] S. Dai, "Comment on "toward complex fuzzy logic"," IEEE Transactions on Fuzzy Systems, p. 1, 2019.

[31] S. Dai, "Complex fuzzy ordered weighted distance measures," Iranian Journal of Fuzzy Systems, 2020.

[32] D. Ramot, M. Friedman, G. Langholz, and A. Kandel, "Complex fuzzy logic," IEEE Transactions on Fuzzy Systems, vol. 11, no. 4, pp. 450-461, 2003.

[33] B. Hu, L. Bi, and S. Dai, "The orthogonality between complex fuzzy sets and its application to signal detection," Symmetry, vol. 9, no. 175, 2017.

[34] L. Bi, B. Hu, S. Li, and S. Dai, "The parallelity of complex fuzzy sets and parallelity preserving operators," Journal of Intelligent \& Fuzzy Systems, vol. 34, no. 6, pp. 4173-4180, 2018.

[35] L. Bi, Z. Zeng, B. Hu, and S. Dai, "Two classes of entropy measures for complex fuzzy sets," Mathematics, vol. 7, no. 96, 2019.

[36] G. Zhang, T. S. Dillon, K.-Y. Cai, J. Ma, and J. Lu, "Operation properties and," International Journal of Approximate Reasoning, vol. 50, no. 8, pp. 1227-1249, 2009.

[37] A. U. M. Alkouri and A. R. Salleh, "Linguistic variables, hedges and several distances on complex fuzzy sets," Journal of Intelligent \& Fuzzy Systems, vol. 26, no. 5, pp. 2527-2535, 2014.

[38] J. Ma, G. Zhang, and J. Lu, "A method for multiple periodic factor prediction problems using complex fuzzy sets," IEEE Transactions on Fuzzy Systems, vol. 20, pp. 32-45, 2012.

[39] L. Bi, S. Dai, and B. Hu, "Complex fuzzy geometric aggregation operators," Symmetry, vol. 10, p. 251, 2018.

[40] L. Bi, S. Dai, B. Hu, and S. Li, "Complex fuzzy arithmetic aggregation operators," Journal of Intelligent \& Fuzzy Systems, vol. 36, pp. 2765-2771, 2019.

[41] S. Greenfield, F. Chiclana, and S. Dick, "Interval-valued complex fuzzy logic," in Proceedings of the 2016 IEEE International Conference on Fuzzy Systems (FUZZ-IEEE), pp. 2014-2019, Melbourne, Australia, December 2016.

[42] S. Greenfield, F. Chiclana, and S. Dick, "Join and meet operations for interval-valued complex fuzzy logic," in Proceedings of the 2016 Annual Conference of the North American Fuzzy Information Processing Society (NAFIPS), pp. 1-5, El Paso, TX, USA, October 2016.

[43] A. Alkouri and A. Salleh, "Complex intuitionistic fuzzy sets," in Proceedings of the International Conference on Fundamental and Applied Sciences (ICFAS 2012), pp. 464-470, Kuala Lumpur, Malaysia, June 2012.

[44] D. Rani and H. Garg, "Complex intuitionistic fuzzy power aggregation operators and their applications in multicriteria 
decision-making," Expert Systems, vol. 35, no. 6, Article ID e12325, 2018.

[45] H. Garg and D. Rani, "Some generalized complex intuitionistic fuzzy aggregation operators and their application to multicriteria decision-making process," Arabian Journal for Science and Engineering, vol. 44, pp. 2679-2698, 2019.

[46] O. Kosheleva, V. Kreinovich, and T. Ngamsantivong, "Why complex-valued fuzzy? Why complex values in general? a computational explanation," in Proceedings of the 2013 Joint IFSA World Congress and NAFIPS Annual Meeting (IFSA/ NAFIPS), pp. 1233-1236, Edmonton, Canada, June 2013.

[47] S. Dai, L. Bi, and B. Hu, "Distance measures between the interval-valued complex fuzzy sets," Mathematics, vol. 7, p. 549, 2019.

[48] E. P. Klement, R. Mesiar, and E. Pap, Triangular Norms, Kluwer Academic Publishers, Dordrecht, Netherlands, 2000.

[49] M. Grabisch, J. L. Marichal, R. Mesiar, and E. Pap, Aggregation Functions, Cambridge University Press, Cambridge, UK, 2009.

[50] M. Grabisch, J. L. Marichal, R. Mesiar, and E. Pap, “Aggregation functions: means," Information Sciences, vol. 181, pp. 1-22, 2011.

[51] G. Choquet, "Theory of capacities," Annales de l'institut Fourier, vol. 5, pp. 131-295, 1954.

[52] M. Sugeno, Theory of fuzzy integrals and its applications, $\mathrm{PhD}$ thesis, Tokyo Institute of Technology, Tokyo, Japan, 1974. 